\title{
Communications
}

2018; 6(1): 5-12

http://www.sciencepublishinggroup.com/j/com

doi: $10.11648 /$ j.com. 20180601.12

ISSN: 2328-5966 (Print); ISSN: 2328-5923 (Online)

\section{Radio Propagation Prediction for HF Communications}

\section{Courage Mudzingwa, Albert Chawanda}

Department of Applied Physics \& Telecommunications, Midlands State University, Gweru, Zimbabwe

Email address:

mudzingwac@gmail.com (C. Mudzingwa),albert.chawanda@gmail.com (A. Chawanda)

\section{To cite this article:}

Courage Mudzingwa, Albert Chawanda. Radio Propagation Prediction for HF Communications. Communications. Vol. 6, No. 1, 2018 , pp. 5-12. doi: 10.11648/j.com.20180601.12

Received: December 30, 2017; Accepted: February 6, 2018; Published: February 27, 2018

\begin{abstract}
The refraction and apparent reflection of HF radio waves by the ionosphere enables long range HF radio communications. The ionosphere is a distinctly irregular medium that is mostly driven by solar activity. Ionospheric models are useful in the prediction of ionospheric behaviour and in the provision of data required for the analysis and forecasting of ionospheric propagation. This paper provides a compact review of HF radio propagation prediction techniques and approaches for HF communications. The paper also highlights the numerous approaches have been used to date in an attempt to estimate F2 usable frequencies. The review presented in this paper is inspired by the most recent advances in the field of ionospheric prediction and modelling.
\end{abstract}

Keywords: HF Communications, Propagation Prediction, Ionosphere, Usable Frequency, MUF

\section{Introduction}

The forecasting of radio wave propagation is considered as an applied science. However, for the past four to eight decades, models for long term forecasting of the median monthly conditions for HF radio propagation have represented an important tool not only for applied science and radio science but also for geophysical researchers in their theoretical studies of the upper atmosphere [1-3]. The main aim of radio propagation forecasting is not only to increase knowledge but rather to improve communication systems. $\mathrm{HF}$ communication is used for short and long range tactical and strategic military purposes since its antennas and equipment can be deployed rapidly to provide immediate command post communications without the need for careful site planning, as is the case with line of sight (LOS) communications. In civilian society, HF is used for international broadcasts by organizations such as the British Broadcasting Corporation and the Voice of America [3]. In Southern Africa, HF exploitation is relatively common and is a primary method for communication since satellite communication infrastructure is not as well improved as in the developed countries. As a result, the use of HF communication is preferred due to its relative simplicity, its capability to provide long range communication at low power without repeater base stations, its ease of development and its low cost [4].

The main purpose of radio propagation forecasting is to give advice in advance about the future reliability of frequency bands propagated by means of the ionosphere. This task is referred to as long-term prediction/forecasting and can be split into a geophysical one, forecasting of a model ionosphere, and one of optical-electromagnetic theory, resolution of the propagation problem [2]. The information about the ionosphere may be called input; the prediction produced in the process is the output. One uses simplified models for the ionosphere and for propagational phenomena, selecting those features of both which have greater influence. Generally there is no unique solution of the forecasting problem; the right effort has to be found in a compromise between the needs of the user or client and the available resources $[2,5]$.

\section{Ionospheric HF Propagation}

The ionosphere is an ionised region of the Earth's upper atmosphere which ranges from about $50 \mathrm{~km}$ to about 1000 $\mathrm{km}$. The extent of ionospheric refraction depends on the density of ionization of the layer, the frequency of the radio wave and the angle at which the wave enters the layer [6]. In order to successfully propagate radio waves through the ionosphere, the frequency cannot be too small, as the wave would then be absorbed, nor too high, for reflection would no 
longer be possible in this case. The two frequencies thus defined are referred to as the lowest usable frequency (LUF) and the maximum usable frequency (MUF) respectively.

The MUF is determined by the degree of ionisation in the ionospheric layer, while the LUF is generally determined by the multihop path attenuation and the noise level at the receive site. The frequency of optimum transmission (FOT) or optimum working frequency (OWF) is the frequency with maximum availability and minimum path loss, and it is generally taken as a percentage of the MUF [5]. The incident angle at which a radio wave enters the ionosphere determines the extent to which that particular wave and the maximum distance that may be covered in a single hop is $4000 \mathrm{~km}$.

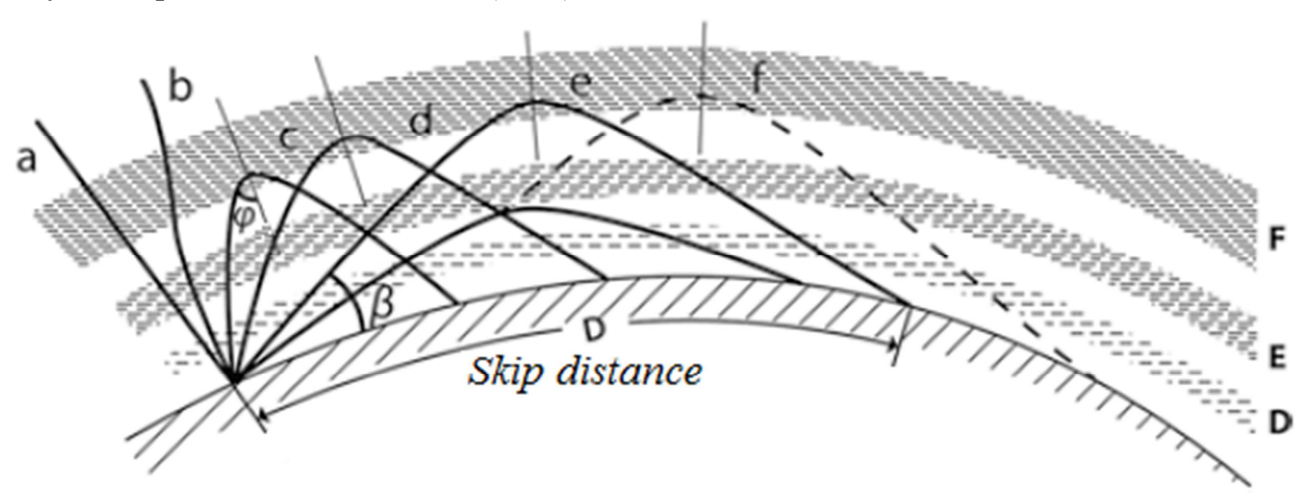

Figure 1. Simplified scheme of an HF radio link [1].

Considering application and operational reasons, HF propagation is basically split into skywave, groundwave and near vertical incidence (NVI). Groundwave propagation is used for relatively short links over a few tens of kilometres whereas skywave propagation can be used worldwide, in suitable ionospheric conditions. Groundwave propagation is affected by the earth's conductivity while skywave propagation is dependent on ionospheric conditions and the effects of the sun. NVI propagation is used for short range HF radio communication. The antenna plays a critical role in NVI propagation by radiating its main beam at a very high take off angle (TOA). The ionosphere is important to skywave radio propagation and provides the basis for almost all $\mathrm{HF}$ communications beyond LOS [7-9]. The ionosphere is also essential in optimising satellite communication systems since the satellite signals traverse the ionosphere, leading to attenuation, depolarization, refraction and dispersion as a result of scattering and frequency dependent group delay. When an HF radio wave reaches the ionosphere, it can be refracted such that it radiates back toward the Earth at some horizontal distance beyond the horizon (see Figure 1). This effect is due to refraction but it is often apparently considered to be a reflection, following Bouguer's refraction law [10]. Figure 1 shows a simple scheme of an HF radio link demonstrating that the behaviour of different electromagnetic rays depends on the angle of elevation $\beta$. In Figure 1 rays $a$ and $b$, with a small angle of incidence $\varphi$ to the ionosphere, escape into space, rays $c, d, e$, and $f$, with an angle of incidence greater or equal than $\varphi$, are reflected by the ionosphere. Intercontinental broadcasting and communication on $\mathrm{HF}$ bands are achieved through ionospheric propagation using the skip phenomenon (as illustrated in Figure 1). Rays reflected by the ionosphere will arrive at greater distances until the elevation angle $\beta$ is tangential to the ground. The skip distance, also known as the silent zone, is the minimum distance that a ray coming back from the ionosphere is reflected. Within the skip distance, only ground wave propagation is possible [7-9, 11].

With increasing antenna take off angle (TOA), $\tau$ (from Figure 2), refracted penetration occurs and the signal is not reflected back to earth. A lower TOA will increase the distance of the first reflection off the F layer of the ionosphere [11]. This increased distance will enhance long-range signal propagation. However, all around the transmitter, a skip zone exists where no reflected rays are received [2].

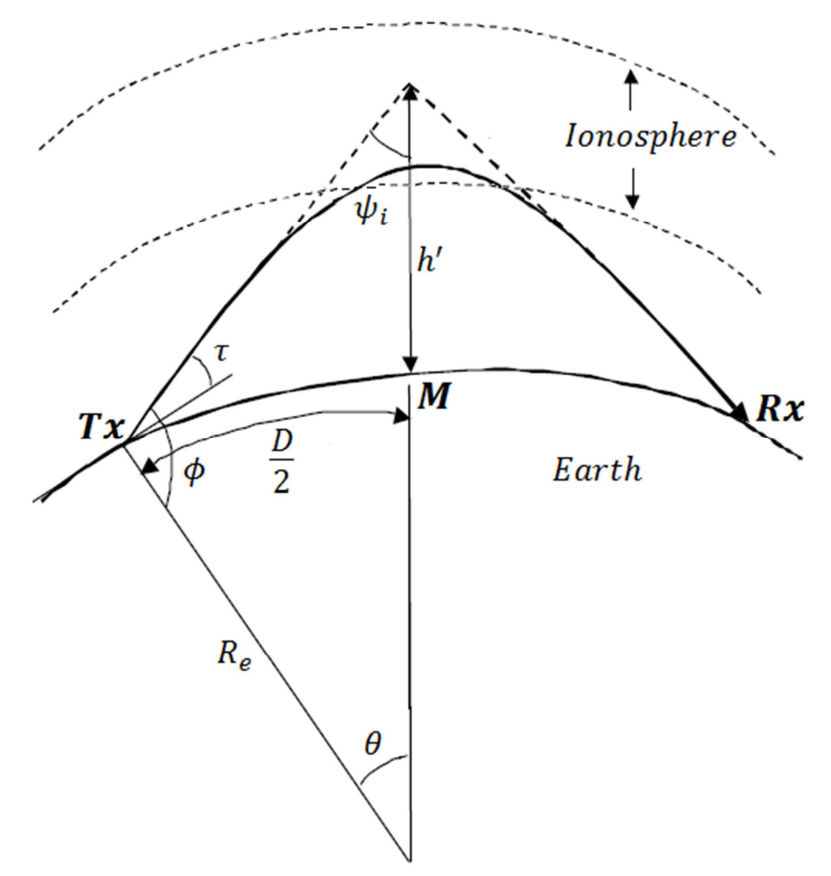

Figure 2. True (solid line) and equivalent (dashed line) trajectories of HF radio waves between two points on Earth through skip mode.

In 1931 Plendl explained the effects of the 11 year solar cycle variation on HF communications [12]. By 1939 it was already known that diurnal conditions also greatly influenced 
the ionospheric communications [13]. In 1937 Smith developed a prediction method of determining the virtual reflection height, for a given frequency $f$, of a triangular path covering the given transmission link ( $\mathrm{Tx}$ to $\mathrm{Rx}$, as indicated in Figure 2) [14]. Smith assumed isotropic dispersion, plane geometry and applied Snell's refraction law [15]. For a curved ionosphere and curved earth, ignoring the effects of the Earth's magnetic field the refractive index $n$ of the ionosphere is given by: [16].

$$
n^{2}=1-\left(\frac{f_{p}}{f}\right)^{2}
$$

where: $f$ is the wave frequency and $f_{p}$ is the plasma frequency. Using the layered approximation to the ionosphere to predict the ray bending, thus a ray entering the ionosphere at an angle of incidence $\psi_{i}$ will be reflected at a height where the ionisation is such that $n$ has the value $[17,18]$ :

$$
n=\sin \psi_{i}
$$

At vertical incidence the reflection condition occurs when $n$ equals zero and from (1) this occurs where $f=f_{p}$. If $f=f_{v}$ represents the vertically incident frequency reflected at the level where the plasma frequency is $f_{p}$ then for the obliquely incident wave:

$$
\sin ^{2} \psi_{i}=1-\left(\frac{f_{p}}{f}\right)^{2}=1-\left(\frac{f_{v}}{f}\right)^{2}
$$

Therefore:

$$
f_{o b}=f_{v} \sec \psi_{i}
$$

Thus an oblique frequency $f_{o b}$ incident on the ionosphere at an angle $\psi_{i}$ will be reflected from the same true height as the equivalent vertical incidence frequency hence a given ionospheric layer will always reflect higher frequencies at oblique incidence than at vertical incidence [7-9]. When $\sec \psi_{i}$ has its maximum value, the frequency $f$ is called the maximum usable frequency (MUF), hence:

$$
M U F=k f_{v} \sec \psi_{i}
$$

Since $\sec \psi_{i}$ changes as the ionosphere changes, it is therefore sufficiently accurate to introduce a correction factor $k$ (Smith's coefficient) so that the secant law in (4) becomes (5) $[14,15,19,20]$. The constant $k$ is a function of path length $(D)$ and reflection height $\left(h^{\prime}\right)$.

From Figure 2, using the law of sines of triangles, $\psi_{i}$ in (5) can be expanded to: [21]

$$
\psi_{i}=\arctan \left[\frac{\sin \left(D / 2 R_{e}\right)}{1+{ }^{h^{\prime}} / R_{e}-\cos \left(D / 2 R_{e}\right)}\right]
$$

where:

$$
\theta=D / 2 R_{e}
$$

$\psi_{i}$ - ray incidence angle, $h^{\prime}$ - virtual height, D - distance between $\mathrm{Tx}$ and $\mathrm{Rx}, \mathrm{M}-$ path midpoint, $R_{e}$ - Earth's radius and $\tau$ - ray take-off angle.

$$
M U F=k f_{v}\left[1+\frac{\sin ^{2}\left(D / 2 R_{e}\right)}{\left[1+h^{\prime} / R_{e}-\cos \left(D / 2 R_{e}\right)\right]^{2}}\right]^{\frac{1}{2}}
$$

Equation (5) describes the simplest $\mathrm{HF}$ ray tracing technique also known as virtual ray tracing [7-9, 16]. Equation (5) basically assumes that the actual propagation can be estimated by reflection from a simple mirror at an appropriate height [22]. This concept is formalised in the secant law, Breit \& Tuve's theorem and Martyn's theorem $[14,15]$. This HF propagation prediction technique has been implemented in various algorithms due to its high computational efficiency. It is usually considered an approximate technique due to its limitations when dealing with a horizontally non-stratified ionosphere [23]. Virtual techniques find their applications in median ionospheric databases and simplified ionospheric profiles. However, when highly accurate and realistic ray tracing are required, in applications such as single site location (SSL) and HF direction finding, numerical and analytical ray tracing techniques are employed [23, 24]. Such techniques are computationally intensive to execute [24-27]. Not all radio propagation prediction programs utilise a ray tracing algorithm, some use virtual geometry techniques whereas others base their predictions on a database of actual forward sounding.

\section{HF Radio Propagation Prediction: Purposes \& Approaches}

Because of spatial and temporal variations of the ionosphere, it is necessary to have detailed knowledge of this variability in order to select the optimum frequency, required transmitter power, antenna specifications, e.t.c. Before the design implementation and confirming planning of radio communication systems, accurate propagation characteristics of the communication path should be well known [16]. In the absence of propagation predictions, such parameter estimations can only be achieved by field measurements which tend to be expensive and time consuming. The formulation of dependable propagation prediction techniques is of profound importance in the advancement of radio science and such techniques can be of engineering value. The purpose of radio propagation prediction is often to ascertain the likelihood of suitable performance of a communication system that is dependent upon electromagnetic wave propagation. This is essential in communication network planning. Ionospheric propagation conditions are certainly variable in space and time, thus, different prediction techniques should be developed according to the duration chosen for the forecast $[18,28,29]$. Basically there are three types of HF propagation classes: long term, medium term and short term, depending on the period on which they are established [16, 30, 31]. Long-term predictions are valid over a period of a month and are developed from ionospheric characteristics, from the prediction of the solar activity index and from statistics of the values of the ionospheric indices 
measured during previous similar situations [32]. Long-term predictions are useful for frequency management, circuit and service planning as well as radio system design and testing. This type of prediction has an important role in the provision of information on the choice of frequency range, Tx location, Tx power and the selection of suitable Tx and Rx antennas. Medium-term predictions are intended at forecasting the general propagation conditions and particularly the MUF values during the next week period [30]. Such predictions are meant for the correction and adaptation of long-term ionospheric forecasts with respect to season as well as solar and geomagnetic activities. The fundamental characteristics of these predictions are therefore their more accurate approximation of seasonal variations and their better account of solar and magnetic activities. On the other hand, short-term predictions are generally stipulated over the next 24 hours and are intended at forecasting the usable frequency band over six hour periods in comparison with the usable frequency band defined over the long term. These short-term predictions are meant for providing corrections of long-term forecasts on a daily basis over permanent areas [33]. As a result, they generally refer to departures from the median behaviour. Short-term frequency predictions are required by the circuit operator in order that he may be able to anticipate MUF failure and thus increase the circuit reliability. Short-term ionospheric fluctuations may be specified in terms of hourly, daily and weekly variabilities. There are also second-to-second and minute-to-minute variations but this group of variations broadly falls within the sphere of unpredictable behaviour. These very short-term predictions are generally referred to as nowcasts [34]. The most common ionospheric short-term fore-casting methods at present mainly include auto-correlation function method, multiple linear regression method, artificial neural network method, equivalent sunspot number method, Kalman filtering method, similar day method, storm time ionospheric forecasting method and so on [35-37].

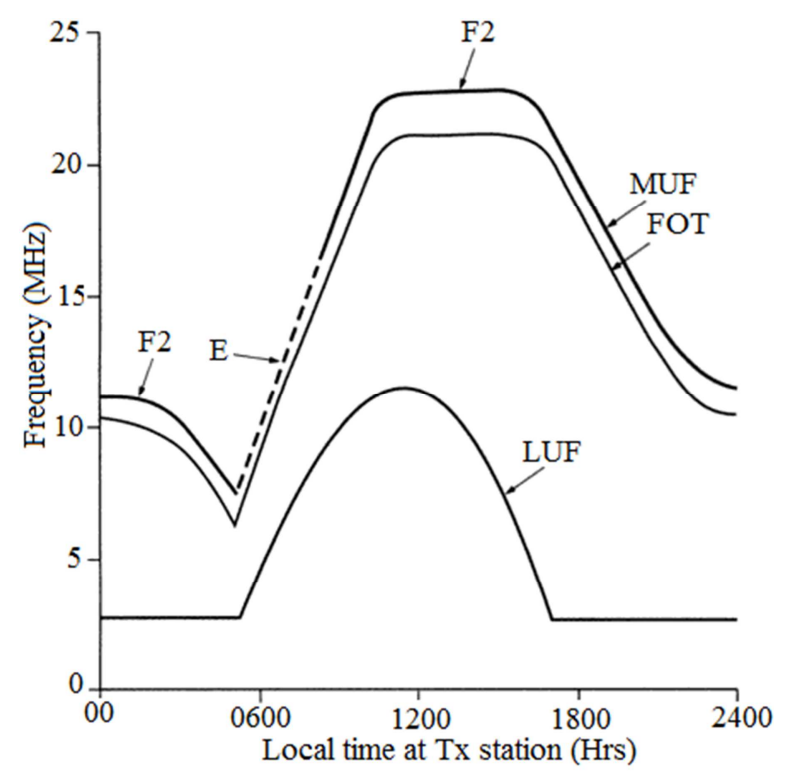

Figure 3. Variations in LUF, MUF and FOT over a 24-hour period.
Figure 3 shows a typical variation of LUF, MUF and FOT over a day. This diagram is indicative only; in practice, it will vary from location to location and between successive days, weeks and months [28]. Apart from the reliance on the geographic location of Tx and Rx, HF propagation conditions of an ionospheric transmission link between two points on the earth vary with daytime, season, and the solar cycle. As a result, it is important to predict the parameters for optimum operational frequencies and other radio propagation applications. Such parameters include the MUF, LUF, FOT and TOA. In the provision of ionospheric services, prediction of ionospheric conditions through time and space has always been a major component. It has been well documented that for $\mathrm{F}$ region modelling the critical frequency of the F2 layer foF2 and its propagation factor M (3000) F2, depend systematically on measurable quantities closely related to solar radiation [22, 38-40]. Ionospheric and solar indices generated from both ionospheric and solar data provide a quick and proxy measure of the complex ionospheric response to variations in solar activity and hence they are useful in mapping the temporal variation of the ionospheric parameters relevant to propagation prediction. On the other hand, the F1 and E layers have a less complex morphology and Chapman's approximations provide an adequate temporal and spatial description for the same purposes [41].

\section{F2 Usable Frequency Prediction}

The F2 layer is considered as the principal ionospheric layer, which also determines the long-range propagation of HF radio waves. Over the years, ionospheric prediction and modelling under specific geophysical conditions has proved to be complex. Usable frequency prediction in HF communications is the foundation of high frequency management. In planning the best use of a given radio communication link, it is important to know in advance what frequencies can be used so as to counter the variability of the ionosphere. Choosing the correct frequency is fundamental to maintaining acceptable communications. The successful choice of the most appropriate frequency depends on the ability to predict and respond to the prevailing ionospheric conditions.

\subsection{MUF Prediction}

It is usually desirable for HF transmission to occur on a frequency as near to the maximum usable frequency over the path at any instant as is feasible under conditions prevailing in practice. We therefore need to know the MUF. In the early days of ionospheric communications, the term "maximum usable frequency" was used without regard to the meaning of "usable" [16, 42]. To standardise the MUF definition, the International Radio Consultative Committee (CCIR) adopted the following recommendation: (i) Basic $M U F$ is the highest frequency of propagation between two points of an HF radio link established only by ionospheric reflection and permitted by the geophysical conditions along the radio path. (ii) Operating $M U F$ is the highest frequency that permits a radio link between two points not only under ionospheric and 
geophysical conditions but also under given working conditions, like class of service, or signal-to-noise ratio, and including the hardware specifications like transmission power and gain of the antenna used $[43,44,45,46]$. In predicting the MUF, there are basically two stages. First, some information is needed about the electron height distribution, $N(h)$. This depends on time of day, time of year, position on earth and sunspot number. A possible method for this is to use a model ionosphere such as the International Reference Ionosphere (IRI) or other regional model ionospheres such as the South African Ionospheric Model (SAIM), in the case of Southern Africa [47, 48]. Second, this information is used to estimate MUF for given positions of the transmitter and receiver [49]. Earlier techniques of predicting MUF involved the use of transmission curves [16]. In this method, MUF is calculated directly from the ionograms obtained during vertical incidence soundings.

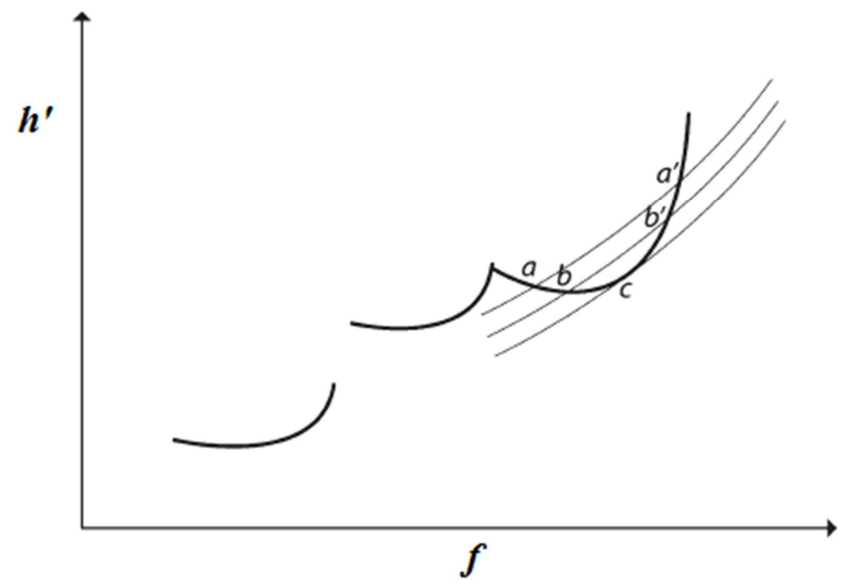

Figure 4. Family of transmission curves parametric in frequency for a fixed distance superimposed on a $h^{\prime} f$ curve [16].

Using the two (4) and (5) it is possible for a given oblique frequency $f_{o b}$ to draw a curve as a function of virtual height, $h^{\prime}$ and $f_{v}$ known as the transmission curve, introduced for the first time by N. Smith in 1939. The intersections of this curve with the curve of the function $h^{\prime}=f\left(f_{v}\right)$ of the ionogram, gives graphical solutions to the two equations, representing the two virtual heights of reflection of a transmission with two propagation paths, points $a$ and $a^{\prime}$ as shown in Figure 4. Then, by increasing the given oblique frequency a family of transmission curves can be obtained, which intersect the ionogram curve at increasingly close points $b$ and $b^{\prime}$, until they become tangential as in point $c$. At this point only one propagation path is possible and the oblique frequency, corresponding to the tangential transmission curve, is the maximum usable frequency for that distance [1, 16, 49]. An in-depth discussion on the status of ionospheric modelling in the context of HF communication systems has been given by [4].

\subsection{FOT and LUF Prediction}

In practice, the HF communications system operator will often not have full knowledge of critical frequencies in real time and at the same time predictions of MUFs are unlikely to be exact, especially when the F2 region is involved. The forecast of solar activity may not be correct. More significantly, the data on which the computation of critical frequencies and effective heights are usually based are averages for periods of a month, whereas ionospheric conditions vary from day to day. It is possible to make allowance for this variability by reducing the calculated operational MUF by an appropriate percentage, producing the FOT, estimated to be the frequency the MUF will exceed on $90 \%$ of the days of the month [50]. FOT is usually the most effective frequency for ionospheric reflection of radio waves between two specified points on Earth and it is usually given by (9). However, some researchers suggest that the value of FOT is usually between $50 \%$ and $80 \%$ of the related MUF [7, 8, 20,30]. The main objective in HF propagation is to keep the Tx frequency as close to the FOT as possible so as to minimise atmospheric absorption but yet not too close to the MUF to lessen ordinary to extraordinary ray fading.

$$
F O T=0.85 M U F
$$

To complete the prediction of usable frequencies, it is necessary to take into account the lower limit. If the operating frequency is reduced, the level of non-deviative absorption in the $\mathrm{D}$ region increases wherever the path of the wave is sun lit. There is also a general tendency for the level of atmospheric noise, the dominant source of noise at HF, to rise at the receiver, with reduction of operating frequency. Thus, for given operating conditions and parameters and for defined channel performance objectives, there will be a LUF. Determination of the LUF is complex since it requires field strength estimation, Tx power, feeder and detuning losses as well as antenna efficiencies [51].

\section{Propagation Prediction Models}

Numerous HF propagation prediction techniques have been used to predict usable frequencies, over long term and short term conditions. These prediction techniques rely on different ionospheric mapping models that derive the key ionospheric characteristics foF2 and M (3000) F2 for radio propagation. Numerous ionospheric mapping methods exist to predict foF2 and M (3000) F2 parameters at any point on the globe or region at any given time. If the mapping method is a long-term prediction of ionospheric characteristics based on monthly median statistics, the prediction will give monthly median values of foF2 and M (3000) F2 for each hour and then subsequentially, the monthly median MUF. On the contrary, if the ionospheric mapping method is a forecast or a nowcast then short-term forecasting or real-time MUF estimation is attained.

\subsection{Long Term Prediction Models}

Various propagation prediction models exist in the form of software to predict the propagation path loss at different frequencies as well as the MUF, FOT, LUF and TOA. Typical input parameters for such prediction programmes include time of day, month, transmitter and receiver position, frequency, sunspot number and possibly a geomagnetic index. The 
geomagnetic index is used in programmes which use special models for high latitudes. Due to the variations and uncertainty in ionospheric conditions, prediction models can only give statistical information. ICEPAC is an enhanced IONCAP (Ionospheric Communications Analysis and Prediction) model developed by the Institute of Telecommunications Sciences (ITS) in Boulder, Colorado, during the 1970s [52]. ICEPAC is a full system performance model for HF radio communications circuits in the frequency range of 2 to $30 \mathrm{MHz}$. It was designed to predict HF sky wave system performance and analyse ionospheric parameters. ICEPAC was developed with a much more elaborate high-latitude ionospheric model called Ionospheric Conductivity and Electron Density (ICED), taking the geomagnetic Q index as an additional input parameter. The computer programme is an integrated system of subroutines designed to predict HF sky wave system performance and analyse ionospheric parameters. ICEPAC predictions require user-defined input data, such as the year, month, day, frequency of operation, type of antenna used, the transmitter power, the type of propagation prediction required and the man-made noise environment, among others [53]. The Australian Government IPS Radio and Space Services HF propagation prediction method is known as the Advanced Stand Alone Prediction System (ASAPS) and is able to predict sky wave radio communication conditions for both the HF and VHF radio spetra. ASAPS was developed by the IPS Radio and Space Services of the Australian Bureau of Meteorology, merges the features of the original IPS method with the ITU-R/ International Radio Consultative Committee (CCIR) models. Numerous graphic representations are based on this method according to the different needs of clients. The graphs have regional or even global validity. The Simplified Ionospheric Regional Model \& Lockwood (SIRM\&LKW) is a monthly median MUF prediction method for a point to point radio link. SIRM\&LKW is based on an empirical method supported by the Istituto Nazionale di Geofisica e Vulcanologia (INGV) for ionospheric HF propagation prediction conditions over the Mediterranean area. In SIRM\&LKW, the predicted foF2 and M (3000) F2 are derived from the monthly median SIRM and foE input values come from the Chapman model [54]. Previous research has confirmed that usable frequencies of an $\mathrm{HF}$ communications link can be predicted, with considerable accuracy, using existing HF propagation prediction models such as REC533 and VOACAP. Various fundamental approaches have also been established for handling the short-term ionospheric variability in HF propagation prediction. Good examples, with varying levels of sophistication, can be found in HF propagation tools such as: HF-EEMS [55] and OpSEND [56] while others are still in development. Extensive research on propagation prediction techniques has been covered by $[1,57-59]$.

\subsection{Nowcasting Models}

Nowcasting refers to merging ionospheric models with real time or near real time foF2 and M (3000) F2 observations, providing users with real time or near real time maps as well as an accurate representation of prevailing ionospheric conditions. The intergration of prediction models and real-time ionospheric measurements in the development of lucid regional or global maps, in line with recent observations, is the basis for nowcasting. The number of nowcasting models has increased in recent years, this includes the SIRM Updating Method \& Lockwood (SIRMUP\&LKW), Instantaneous Space Weighted Ionospheric Regional Model (ISWIRM) and SAIM, for the Southern African region. SIRMUP\&LKW model predicts the daily hourly MUF values by using the nowcasting SIRMUP model to derive foF2 and M (3000) F2 parameters [60]. ISWIRM is a regional foF2 nowcasting model, applicable within the geographic range between $35^{\circ}$ $70^{\circ} \mathrm{N}$ and $51^{\circ} \mathrm{W}-40^{\circ} \mathrm{E}$. Within this geographic region, the hourly values of foF 2 are obtained correcting the monthly medians values of foF2, predicted by the Space Weighted Ionospheric Local Model (SWILM) [61], basing on hourly foF2 observations from four reference ionosondes. For in-depth detail on the development of SAIM, refer to [48].

\section{Limitations to Accurate F2 Usable Frequency Prediction}

The successful prediction of F2 usable frequencies depends on the accuracy of F2 peak parameter prediction, hence limitations to accurate F2 predictions has a cumulative effect on F2 usable frequency predictions. Notable limitations to accurate F2 usable frequency prediction includes:

1. The relationships governing the global distribution of ionospheric parameters as a whole are still not completely understood [62].

2. There is no widely adopted morphology of the F2 region at nighttime, rendering the interpretation of nighttime diurnal variations complex. Since various types of nighttime enhancements in the F2 layer electron concentration manifest, nighttime F2 layer related predictions may be much less accurate than daytime predictions. [63, 64].

3. The unavailability of complete knowledge of the F2 layer in its perturbed state poses major limitations, not only on the prediction of usable frequencies, but also on interpreting the complexities surrounding the spatial and temporal evolution of the perturbation effect. Besides, apart from the beginning of cyclical geomagnetic disturbances, the prediction of temporal variation of disturbances a few hours or more in advance is not yet possible [65].

4. The reliability of ionospheric parameter prediction is determined by precise and dependable knowledge about the evolution of the ionosphere as well as the accuracy and absoluteness of inputs which are used in predictive computations.

\section{Conclusion}

This paper has reviewed and analysed various techniques and approaches that have been used to date in predicting or 
forecasting ionospheric parameters and F2 usable frequencies. The intricacy in the prediction of MUF, FOT, TOA and LUF was also discussed while highlighting the subsequent limitations that affect the accuracy of F2 propagation predictions.

\section{References}

[1] B. Zolesi and L. R. Cander, Ionospheric Prediction and forecasting, Springer, New York, 2014.

[2] K Rawer, The historical development of forecasting methods for ionospheric propagation of HF waves, Radio Sci, vol 10, no. 7, pp. 669-679, 1975.

[3] L. Barclay (ed), Propagation of radio waves, The institution of Engineering and Technology, UK, 2003.

[4] J. M. Goodman, Operational communication systems and relationships to the ionosphere and space weather, Adva. Space. Res., vol. 36, pp. 2241-2252, 2005.

[5] L. F. McNamara, C. R. Baker and W. S. Borer, Real-time specification of HF propagation support based on a global assimilative model of the ionosphere, Radio Sci., vol. 44, doi:10.1029/2008RS004004, 2009.

[6] P. G. Brasseur and S. Solomon, Aeronomy of the middle atmosphere: chemistry and physics of the stratosphere and mesosphere: Third revised and enlarged edition, Springer, Netherlands, 2005.

[7] J. S. Seybold, Introduction to RF propagation, Wiley, New Jersey, 2005.

[8] J. A. Richards, Radio wave propagation, Springer, Germany, 2008.

[9] N. M Maslin, HF Communications, Taylor \& Francis, UK, 2005.

[10] N. Blaustein and C. G. Christodoulou, Radio Propagation and Adaptive Antennas for Wireless Communication Links, Wiley, New Jersey, 2007.

[11] J. J. Carr, Antenna Toolkit: $2^{\text {nd }}$ Edition, Newnes, Oxford, 2001.

[12] H. Plendl, Concerning the influence of the eleven-year solar activity period upon the propagation of waves in wireless telegraphy, Proc. Inst. Radio Eng., vol. 20, Issue 3, pp. 520-539, 1932 .

[13] K. Rawer, Propagation of decameter waves (HF-Band), Meteorological and Astronomical Influences on Radio Wave Propagation, ed. By B. Landmark, pp. 221-250, New York, 1963.

[14] N. Smith, Extension of normal-incidence ionosphere measurements to oblique incidence radio transmission, Journal of Research of the National Bureau of Standards, vol. 19, pp. 89-94, 1937.

[15] N. Smith, The Relation of Radio Sky-Wave Transmission to Ionosphere Measurements, Proc. Inst. Radio Eng., pp. 332-347, 1939.

[16] K. Davies, Ionospheric Radio Propagation, Dover Publications, New York, 1966.
[17] D. C. Jenn, EC3630 Radiowave Propagation, Naval Postgraduate School: Dep. of Elec \& Comp. Eng., California, 2010 .

[18] C. Mudzingwa, A. Nechibvute and A. Chawanda, Maximum Useable Frequency Prediction Using Vertical Incidence Data, Int. Journ. of Eng. Res. and Tech., vol. 2, no. 8, pp. 2050-2056, 2013.

[19] A. G. Kim and G. V. Katovich, Preliminary results for electron density profile reconstruction from weakly oblique sounding data, Proc. of SPIE, vol. 6936, 2008.

[20] A. Ghasemi, A. Abedi, and F. Ghasemi, Propagation engineering in wireless communications, Springer, New York, 2012.

[21] M. Muhlhauser and I. Gurevych, Handbook of research on ubiquitous computing technology for real time enterprises, Information Science Reference, New York, 2008.

[22] L. Barclay (ed.), Propagation of radio waves, The Institution of Engineering and Technology, UK, 2003.

[23] L. F. McNamara, The Ionosphere: Communications, Surveillance and Direction Finding, Krieger Pub. Co., 1991.

[24] R. M. Jones and J. J. Stephenson, A three dimensional ray tracing computer program for radio waves in the ionosphere, US. Dept. of Commerce Office of Telecommunications OT report 75-76, 1975.

[25] J. P. Villain, R. A. Greenwald and J. F. Vickrey, HF ray tracing at high latitudes using measured meridional electron density distributions, Radio Sci., vol. 19, no. 1, pp. 359-374, 1984.

[26] G. Miro' Amarante and S. M. Radicella, Use of ray tracing in models to investigate ionospheric channel performance, Adva. Space Res., vol. 39, pp. 926-931, 2007.

[27] X. Huang and B. W. Reinisch, Real-time HF ray tracing through a tilted ionosphere, Radio. Sci., vol. 41, RS5S47, 2006.

[28] A. Graham, Communications, Radar and Electronic Warfare, Wiley, UK, 2011.

[29] R. D. Hunsucker and J. K. Hargreaves, The high latitude ionosphere and its effects on radio propagation, Cambridge Univ. Press, 2002.

[30] H. Sizun, Radio wave propagation for Telecommunication Applications, Springer, Berlin, 2005.

[31] R. Hanbaba, Perfomance prediction methods of HF radio systems, Annali Di Geofisica, vol. 41, no. 5-6, pp. 715-742, 1998.

[32] COST 238, PRIME (Prediction and Retrospective Ionospheric Modelling over Europe). Final report, Commission of the European Communities, 1999.

[33] COST 251, Improved Quality of Service in Ionospheric Telecommunication Systems Planning and Operation, Final report, Commission of the European Communities, 1999.

[34] J. M. Goodman, Space Weather and Telecommunications, Springer, New York, 2005.

[35] J. Feng, A new method for ionospheric short-term forecast using similar-day modelling, Antennas, Propagation \& EM Theory (ISAPE), $10^{\text {th }}$ International Symposium, pp. 472-474, 2012. 
[36] J. D. Huba, R. W. Schunk and G. V. Khazano (ed.), Modeling the Ionosphere-Thermosphere, AGU, Washington, 2013.

[37] P. P. Ban, S. J. Sun, C. Chen and Z. W. Zhao, Forecasting of low-latitude storm-time ionospheric foF2 using support vector machine, Radio Sci., vol. 46, 2011.

[38] P. A. Bradley, Further study of foF2 and M (3000) F2 in different solar cycles, Ann Geofis, 37:201-208, 1994.

[39] ITU-R SG3, Handbook on ionospheric properties and propagation, Geneva, 1996.

[40] ITU-R Rec. P. 1239, ITU-R Reference ionospheric characteristics, International Telecommunication Union, Geneva, 1997.

[41] S. Chapman, The absorption and dissociative or ionizing effect of monochromatic radiation in an atmosphere on a rotating Earth, Proc. Phys. Soc., vol. 43, pp. 26-45, 1931.

[42] S. Y. Ji, J. Dong and J. Wang, Short-term forecasting method of usable frequency based on vertical sounding data in single station, WIT Transa. on Info. and Comm. Tech., vol. 60, 2015.

[43] ITU-R Rec. P. 373-7, Definitions of maximum and minimum transmission frequencies. International Telecommunication Union, Geneva, 1995.

[44] J. Whithers, Radio spectrum management: management of the spectrum and regulation of radio services, IEE Telecommunications, series 45, London, 1999.

[45] I. Poole, Basic radio: Principles and Technology, Newnes, London, 1998.

[46] J. Wang, Basic MUF observation and comparison of HF radio frequency prediction based on different ionosphere models, IEEE ISAPE, pp. 403-406, 2010.

[47] F. F. Mazda (ed.), Electronics engineers' reference book, $6^{\text {th }}$ Ed., Butterworth-Heinemann Ltd, London, 1989.

[48] D. I. Okoh, L. A. McKinnell and P. J. Cilliers, Developing an ionospheric map for South Africa, Ann. Geophys., vol. 28, pp. 1431-1439, 2010.

[49] K. G. Budden, The propagation of radio waves, Cambridge University Press, Cambridge, 1985.

[50] J. Whithers, Radio spectrum management: management of the spectrum and regulation of radio services, IEE Telecommunications, Series 45, London, 1999.

[51] G. Lane, F. J. Rhoads and L. Deblasio, Voice of America Coverage Analysis Program (VOACAP): A Program Guide, VOA B/ESA Report 01-93, 1993.

[52] ITS, Ionospheric Communications Enhanced Profile Analysis \& Circuit (ICEPAC) prediction program user's manual, Institute for Telecommunication Sciences, Boulder, Colorado, 2007.
[53] L. R. Teters, J. L. Lloyd, G. W. Haydon and D. L. Lucas, Estimating the perfomance of telecommunication systems using the ionospheric channel: (Volume II) Ionospheric Communications Analysis and Prediction Program user's manual, Institute for Telecommunication Sciences NTIA Report 83-127, July 1983.

[54] M. Lockwood, A simple M-factor algorithm for improved estimation of the basic maximum usable frequency of radio waves reflected from the ionospheric $\mathrm{F}$ region, Proceedings of the IEE 130F, pp. 296-302, 1983.

[55] A. K. Shukla, P. S. Cannon, S. Roberts and D. Lynch, A tactical $\mathrm{HF}$ decision aid for inexperienced operators and automated HF systems, 7th International Conference on HF Radio Systems and Techniques, pp. 383, IEE, Nottingham, UK, 1997.

[56] G. Bishop, T. Bullett, K. Groves, S. Quigley, P. Doherty, E. Sexton, K. Scro, R. Wilkes and P. Citrone, Operational Space Environment Network Display (OpSEND), 10th International Ionospheric Effects Symposium, Alexandria, Virginia, USA, 2002.

[57] C. Levis, J. T. Johnson and F. L. Teixeira, Radiowave propagation: Physics and applications, Wiley, 2010.

[58] M. F. Iskander and Z. Yun, Propagation prediction models for wireless communication systems, IEEE Transactions on Microwave Theory and Techniques, vol. 50, no. 3, 2002.

[59] R. Hanbaba, Performance prediction methods of HF systems, Annal. Di Geofisica, vol. 41, no. 5-6, 1998.

[60] B. Zolesi, A. Belehaki, I. Tsagouri and L. R. Cander, Realtime updating of the simplified ionospheric regional model for operational applications, Radio Sci., vol. 39, no. 2, 2004.

[61] M. Pietrella and L. Perrone, Instantaneous space weighted ionospheric regional model for instantaneous mapping of the critical frequency of the F2 layer in the European region, Radio Sci., vol. 40, no. 1, 2005.

[62] J. N, Korenkov, Ionospheric modelling, Springer Basel AG, Germany, 1988.

[63] A. F. Yakovets, V. V. Vodyannikov, G. I. Gordienko and Y. G. Litvinov, Some features of nighttime enhancements in the electron concentration in the F2 layer maximum of the midlatitude ionosphere, Geomagn. Aeron., vol. 54, no. 6, pp. 807-816.

[64] G. Chen, H. Qi, B. Ning, Z. Zhao, M. Yao, Z. Deng, T. Li, S. Huang, W. Feng, J. Wu and C. Wu, Nighttime ionospheric enhancements induced by the occurrence of an evening solar eclipse, Journ. of. Geohys. Research: Space Physics, vol. 118, pp. 6588-6596, 2013.

[65] W. Fengsi, C. Hongchang, F. Xueshang and S. Jiankui, A prediction method of geomagnetic disturbances based on IPS observations-dynamics-fuzzy mathematics, Adva. in Space Res., vol. 31, no. 4, pp. 1069-1073. 